\section{Seroconversion from Anti-Th/To to Anticentromere Antibodies in a Patient with Systemic Sclerosis}

\section{To the Editor:}

Antinuclear antibodies (ANA) are involved in the diagnosis of systemic sclerosis (SSc), being present in $90 \%$ of the patients ${ }^{1,2,3}$. In the majority of ANA-positive patients with SSc, a single SSc-specific autoantibody can be detected, i.e. antibodies to centromere protein B (CENP-B $)^{4}$, topoisomerase $\mathrm{I}^{5}$, RNA Pol-III ${ }^{3}$, and $\mathrm{Th} / \mathrm{To}^{1,2,3,6,7}$. These 4 major autoantibodies are typically mutually exclusive. It has been found that anti-Rpp 25 antibodies are an important target of antibodies to the $\mathrm{Th} / \mathrm{To}$ complex ${ }^{6,7}$. Also, we discovered an epitope located on the Rpp38 subunit of the Th/To complex as the target of autoantibodies in patients with SSc. Anti-Th/To antibodies have been associated with the limited cutaneous form of SSc and with lung disease $^{8}$. Case reports with sequential serum samples are valuable tools to study the evolution of autoantibody responses in autoimmune diseases ${ }^{9}$. We report a case of SSc with seroconversion from anti-Th/To to anti-CENP-B antibodies

The case report was approved by the Research Ethics Board under the registration number BD05.001.

A 58-year-old woman was diagnosed with limited cutaneous SSc in 1994 on the basis of Raynaud phenomenon (onset at $43 \mathrm{yrs}$ ), sclerodactyly, and capillary telangiectases. Ten sequential serum samples were collected between 1993 and 2011. All samples were tested for anti-Th/To antibodies by prototype QUANTA Flash Rpp256,7 and Rpp38 chemiluminescent immunoassays (Figure 1), and for anti-CENP-B, Scl-70, PM/Scl, and RNA Pol III antibodies by QUANTA Flash or ELISA and by QUANTA Flash CTD Screen Plus (as detailed in Figure 2; all Inova Diagnostics). Immunoadsorption with Rpp25-coupled beads was used to demonstrate that anti-Rpp 25 were the only detectable antibodies in the first serum sample (Figure 2).

The first sample (1993) showed a positive result for anti-Rpp25 antibodies as the only detectable antibody (Figure 1), confirmed by immunoadsorption using Rpp25-coupled beads and testing on CTD Screen
Plus. Anti-Scl-70, anti-PM/Scl, and anti-RNA Pol III were negative in all sera of the patient. The second sample obtained 5 years later showed a lower anti-Rpp25 antibodies level, but exhibited seroconversion to high levels of anti-CENP-B antibodies that correlated with onset of a centromere pattern by indirect immunofluorescence on HEp-2 cells (Figure 1, panel B). Subsequent samples, taken while the patient was receiving only nifedipine, showed decreasing anti-CENP-B titers and a more marked decrease in anti-Rpp25 antibodies. In 2006, a thorax computed tomography (CT) scan displayed no sign of interstitial lung disease. Interestingly, the serum sample obtained in October 2009 revealed an increase in anti-CENP-B and onset of antibodies to the Rpp38-derived peptide, which were negative on 6 previous serum samples. In December 2010, cardiac magnetic resonance imaging revealed myocardial fibrosis and moderate pericardial effusion. Pulmonary hypertension and dilatation of esophagus were also reported. The patient was hospitalized in December 2011 for abdominal pain and vomiting. Diffuse small intestinal dilatation was present by CT scan, consistent with small bowel involvement by SSc and pseudo-obstruction. A chest radiogram showed bilateral pleural effusions and pulmonary edema. During the following days, increasing pulmonary infiltrates led to diagnosis of multifocal pneumonia, and parenteral antibiotics were initiated. In April 2012, partial improvement of the infiltrates was noted together with onset of new infiltrates of the right lung base. Fifteen days later, improvement of infiltrates of the right lung base but new infiltrates of the left lung base were recorded. The patient died shortly thereafter of aspiration pneumonia and global heart failure.

To our knowledge, ours is the first reported case of seroconversion from anti-Th/To (Rpp25) to anti-CENP-B autoantibodies in a patient with SSc monitored over 17.5 years. In addition, onset of anti-Rpp38 antibodies was documented later, consistent with epitope spreading. Both anti-CENP-B and anti-Th/To are associated with limited cutaneous SSc, which may be complicated by severe heart and gastrointestinal involvement, consistent with the disease course in this patient ${ }^{10}$. Intriguingly, both anti-CENP-B and antiRpp38 levels rose prior to clinical exacerbation. Although anti-Th/To and anti-CENP-B are usually mutually exclusive, these major SSc autoantibodies may be expressed with fluctuating levels in the same patient.
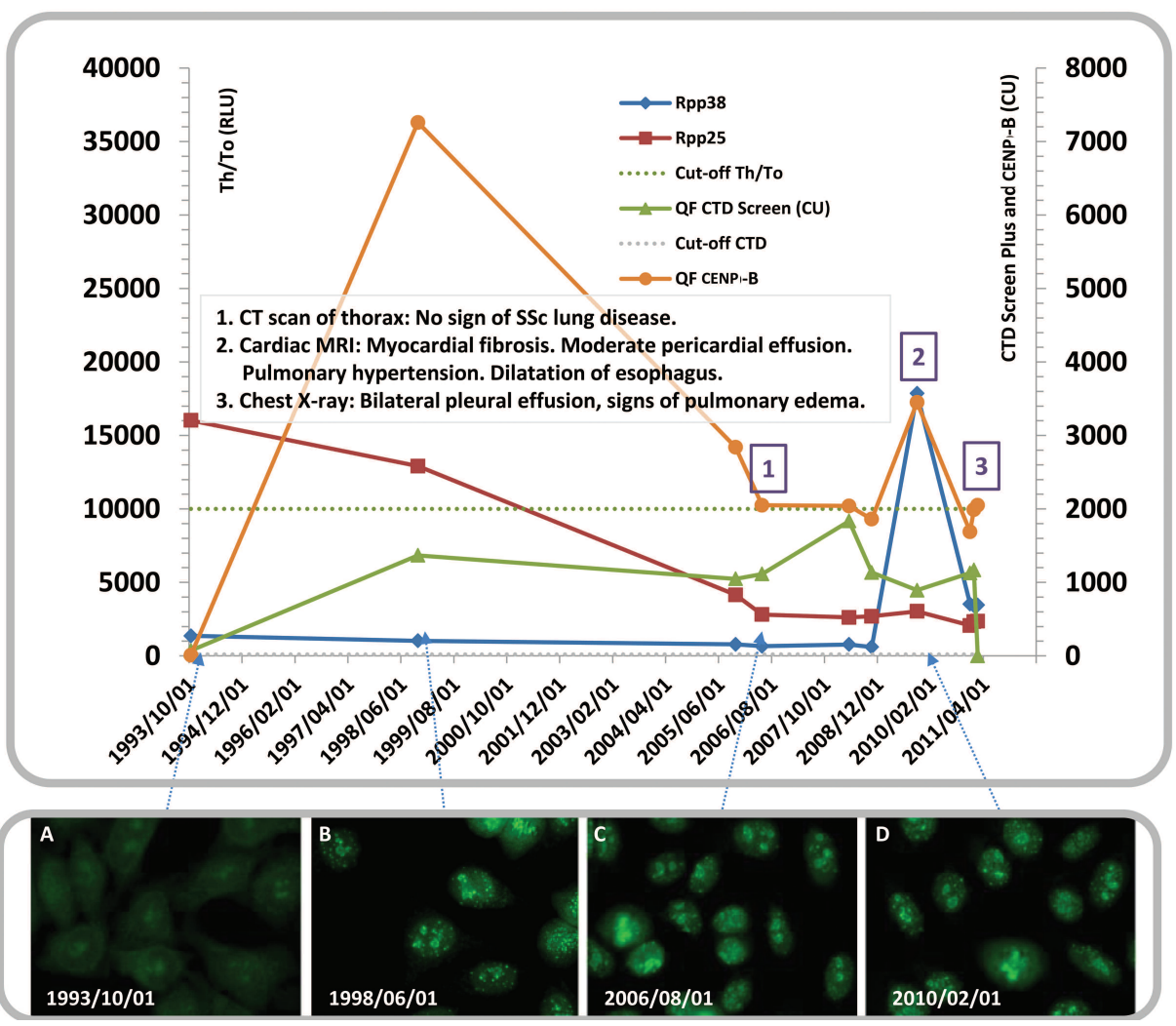

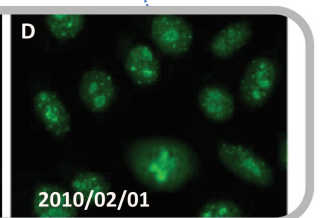

Figure 1. Clinical and serological evolution of the presented case. The antibody titers for the $2 \mathrm{Th} / \mathrm{To}$ antigens (recombinant Rpp25 and the Rpp38 derived peptide) are shown on the left Y-axis and expressed as RLU. Cutoff values for the anti-Rpp38 and anti-Rpp25 assays are 10,000 RLU. On the right Y-axis, the results of all the other assays are displayed. In addition, important clinical events are indicated by the boxed numbers (1-3). Panels A to D are pictures displaying fluorescent antinuclear antibody results by indirect immunofluorescence on HEp-2 cells at the corresponding date, as indicated. RLU: relative light units; CTD: connective tissue disease; CT: computed tomography; SSc: systemic sclerosis; MRI: magnetic resonance imaging; $\mathrm{CU}$ : chemiluminescence units; CENP-B: centromere protein B; QF: QUANTA Flash. 


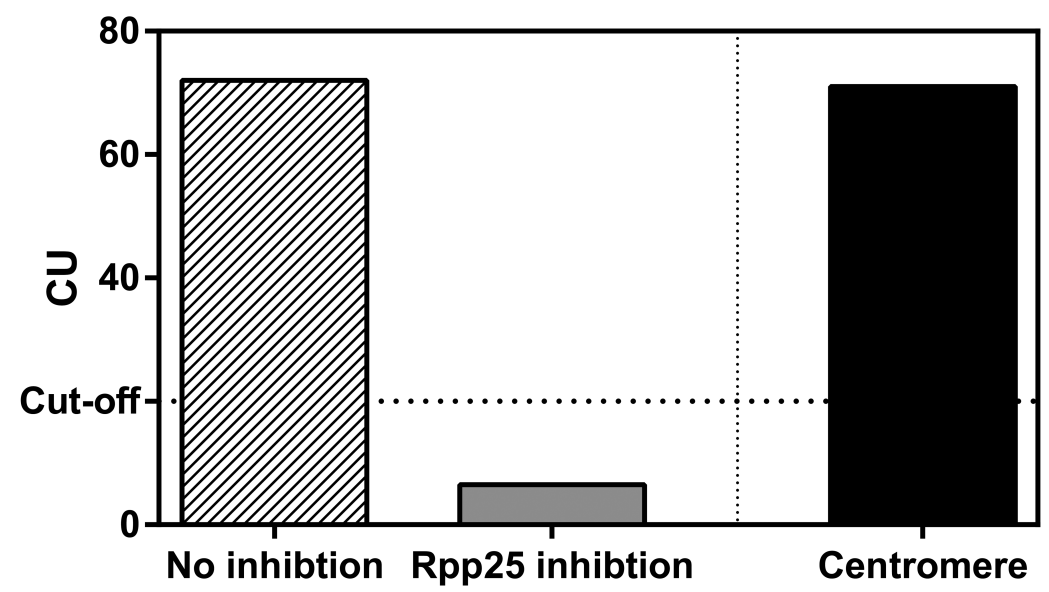

Figure 2. Immunoadsorption of anti-Rpp25 antibodies. The first serum sample of the patient was incubated with paramagnetic beads coupled with recombinant Rpp25 antigen to immunoadsorb antibodies and then tested on QUANTA Flash CTD Screen Plus. As control, beads coupled with recombinant centromere protein B (CENP-B) were used. Inhibition was observed with Rpp25, but not with CENP-B, indicating that anti-Rpp25 antibodies are the only detectable antibody in the first serum sample of the patient. Antigens contained in the QUANTA Flash CTD Screen Plus: dsDNA, Sm/RNP, Ro52, Ro60, SS-B, Scl-70, CENP, $\mathrm{Mi}-2, \mathrm{Ku}, \mathrm{Th} / \mathrm{To}$, RNA Pol III, Pm/Scl, PCNA, Jo-1, and ribosomal-P protein. CTD: connective tissue disease; $\mathrm{CU}$ : chemiluminescence units.

MARTIAL KOENIG, MD, Department of Medicine, University of Montreal Faculty of Medicine, Division of Rheumatology and Autoimmunity Research Laboratory, Research Center of the Centre Hospitalier de l'Université de Montréal; JEAN-LUC SENÉCAL, MD, FRCPC, Department of Medicine, University of Montreal Faculty of Medicine, Division of Rheumatology and Autoimmunity Research Laboratory, Research Center of the Centre Hospitalier de l'Université de Montréal, Montréal, Québec, Canada; MICHAEL MAHLER, PhD, Inova Diagnostics, San Diego, California, USA. Dr. Senécal holds the University of Montreal Scleroderma Research Chair. The research involving this case report is supported by Sclérodermie Québec, Scleroderma Society of Ontario, Scleroderma Society of Canada, and Scleroderma Association of Saskatchewan. Address correspondence to Dr. M. Mahler, Vice President of Research, Inova Diagnostics Inc., 9900 Old Grove Road, San Diego, California 92131-1638,USA.E-mail: mmahler@inovadx.com

\section{REFERENCES}

1. Okano Y, Medsger TA Jr. Autoantibody to Th ribonucleoprotein (nucleolar 7-2 RNA protein particle) in patients with systemic sclerosis. Arthritis Rheum 1990;33:1822-8.

2. Koenig M, Joyal F, Fritzler MJ, Roussin A, Abrahamowicz M, Boire $\mathrm{G}$, et al. Autoantibodies and microvascular damage are independent predictive factors for the progression of Raynaud's phenomenon to systemic sclerosis: a twenty-year prospective study of 586 patients, with validation of proposed criteria for early systemic sclerosis Arthritis Rheum 2008;58:3902-12.

3. Mahler M, Meroni PL, Bossuyt X, Fritzler MJ. Current concepts and future directions for the assessment of autoantibodies to cellular antigens referred to as anti-nuclear antibodies. J Immunol Res 2014;2014:315179.

4. Koenig M, Dieudé M, Senécal JL. Predictive value of antinuclear autoantibodies: the lessons of the systemic sclerosis autoantibodies. Autoimmun Rev 2008;7:588-93.

5. Mahler M, Silverman ED, Schulte-Pelkum J, Fritzler MJ. Anti-Scl-70 (topo-I) antibodies in SLE: Myth or reality? Autoimmun Rev 2010;9:756-60.

6. Mahler M, Gascon C, Patel S, Ceribelli A, Fritzler MJ, Swart A, et al. Rpp25 is a major target of autoantibodies to the $\mathrm{Th} / \mathrm{To}$ complex as measured by a novel chemiluminescent assay. Arthritis Res Ther 2013;15:R50.

7. Mahler M, Satoh M, Hudson M, Baron M, Chan JY, Chan EK, et al. Autoantibodies to the Rpp25 component of the Th/To complex are the most common antibodies in patients with systemic sclerosis without antibodies detectable by widely available commercial tests. J Rheumatol 2014;41:1334-43.

8. Mahler M, Fritzler MJ, Satoh M. Autoantibodies to the mitochondrial RNA processing (MRP) complex also known as Th/To autoantigen. Autoimmun Rev 2015;14:254-7.

9. Mahler M, Mierau R, Genth E, Blüthner M. Development of a CENP-A/CENP-B-specific immune response in a patient with systemic sclerosis. Arthritis Rheum 2002;46:1866-72.

10. Steen VD. Autoantibodies in systemic sclerosis. Semin Arthritis Rheum 2005;35:35-42.

J Rheumatol 2017;44:12; doi:10.3899/jrheum.170575 